\title{
Structural organization and evolution of the marsupial zona pellucida
}

\author{
William G. Breed ${ }^{1}$, Rory M. Hope², Ole W. Wiebkin³, \\ Scott C. Spargo ${ }^{2}$ and Jamie A. Chapman ${ }^{1}$ \\ ${ }^{1}$ Department of Anatomical Sciences, ${ }^{2}$ Laboratory of Molecular Evolution, Department of \\ Molecular Biosciences and ${ }^{3}$ Department of Medicine, The University of Adelaide, Adelaide, \\ South Australia 5005, Australia
}

\begin{abstract}
In this review, the biochemical composition and structural organization of the marsupial and eutherian zonae pellucidae are compared. Differences between the zonae from these two groups of mammals are observed in their response to dilute proteases and reducing agents, in their potential glycosylation patterns, and in some of their functions. However, studies on the glycoconjugates and polypeptides of the three zona pellucida genes have not explained these different responses to the proteases and reducing agents. There is high sequence similarity between the zona polypeptides of marsupials and eutherians, as well as a similarity in the oligosaccharides present, as demonstrated by lectin staining. As the marsupial and eutherian lineages diverged from a common ancestor over 100 million years ago, these observations indicate that the three-dimensional structure of these glycoproteins is highly conserved throughout all mammals, although the complexity of its molecular organization has yet to be resolved. Phylogenetic analyses indicate that there are at least four groups of paralogous zona pellucida genes in vertebrates. The marsupial ZPA and ZPB genes have been named in accordance with their orthologues but the phylogenetic relationships of the marsupial ZPC gene require further investigation.
\end{abstract}

In sexually reproducing organisms a new individual is formed when two haploid cells, the spermatozoon and the egg, come together to form a diploid zygote. In most internally fertilizing species, the spermatozoa are deposited in the lower regions of the female reproductive tract and, from there, migrate up to the higher reaches of the tract where sperm-egg fusion and zygote formation result. Before fusion and zygote formation can occur, the spermatozoon must bind to, and penetrate, an extracellular coat that surrounds the egg. Once binding and penetration have taken place, the spermatozoon can fuse with the cell membrane of the egg, the oolemma, resulting in syngamy and oocyte activation.

An acellular coat around recently ovulated eggs is present in all vertebrates and even around some invertebrate eggs, such as those of sea urchins. In vertebrates, this coat is variously referred to as the chorion in fish, the vitelline envelope in amphibians, the perivitelline envelope in reptiles and birds, and the zona pellucida in both eutherian and marsupial mammals. Although the general morphology of the coat is similar in all of these vertebrate lineages, its thickness varies greatly among the major groups. In marsupials, the zona pellucida tends to be somewhat thinner than in eutherians (Fig. 1) and this feature

Email: bill.breed@adelaide.edu.au may be associated with a more lytic process of zona penetration than that which occurs in eutherian mammals (Bedford, 1991, 1998). In this review, the recently acquired knowledge of the structure and function of the zona pellucida of marsupials is compared with that already documented in eutherian mammals.

Although several functions of the eutherian zona pellucida are fairly well established, the organization of the structural components, and their evolutionary relationships, remain equivocal. It has generally been believed that the eutherian zona pellucida is composed of three sulphated glycoproteins, ZPA (ZP2 in rodents), ZPB (ZP1 in rodents), and ZPC (ZP3 in rodents) (for example, Wassarman, 1988; Dunbar et al., 1994; Epifano et al., 1995, Wassarman et al., 1999). (The terms ZPA, ZPB and ZPC are used in the present review to describe the major glycoprotein components of the zona pellucida as proposed by Harris et al., 1994.) It has been assumed that comparisons between, for example, human and mouse ZPB are comparisons between orthologous genes, that is, genes that trace their origin to a single gene present in the most recent common ancestor. Recent work on laboratory mice and humans indicates that such a view may be oversimplistic and that a gene, named ZP1 to distinguish it from human ZPB, is the true human orthologue of the mouse ZPB (ZP1) gene (Hughes and Barratt, 1999). In the context of these uncertainties and the 


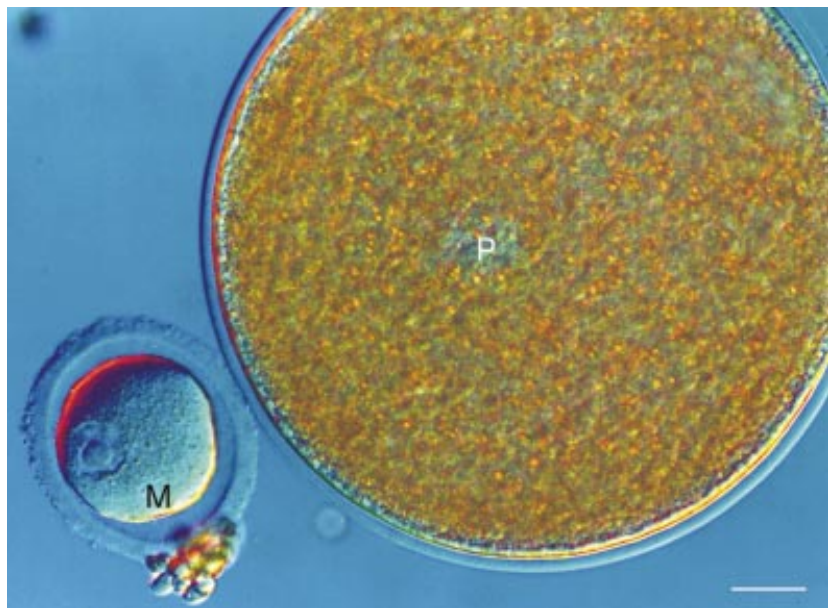

Fig. 1. Nomarski differential optics of an ovarian oocyte from a large follicle of a laboratory mouse (M) and brushtail possum, Trichosurus vulpecula (P). The zona pellucida is thicker around the mouse oocyte than that around the considerably larger possum oocyte. Scale bar represents $25 \mu \mathrm{m}$.

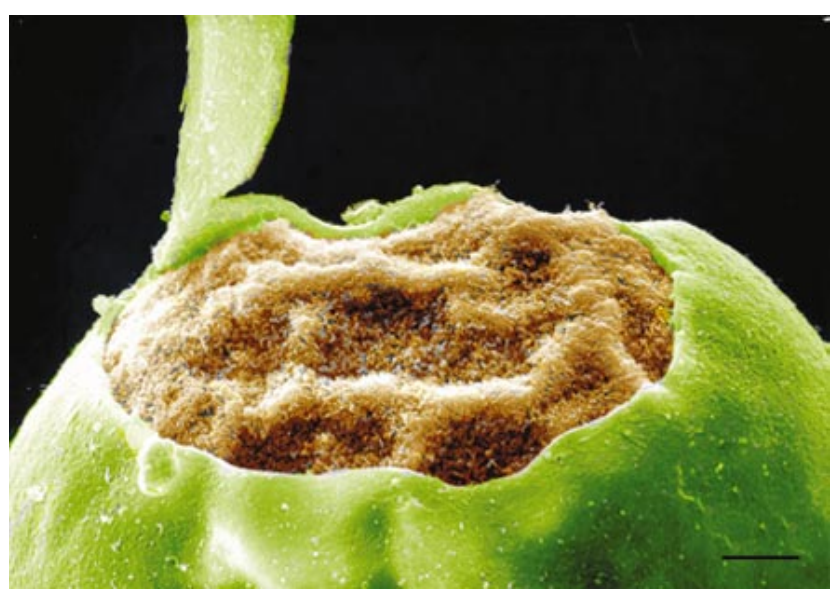

Fig. 2. Scanning electron micrograph of part of a recently ovulated oocyte from the dasyurid marsupial Sminthopsis crassicaudata. The zona pellucida around the oocyte was torn after its attachment to a polylysine-coated coverslip to show the underlying microvilli of the oocyte. Scale bar represents $10 \mu \mathrm{m}$.

resultant confusion in gene and protein nomenclature, comparisons between marsupial and eutherian zona gene homologues may be enlightening. Accordingly, the evolution of the zona pellucida genes in Mammalia are examined and special consideration is taken of the recently published cDNA sequence data from eutherians and marsupials.

\section{Functions of the mammalian zona pellucida}

In eutherian mammals, the zona pellucida has several known functions, including: (i) provision of binding sites for receptors on the sperm surface; (ii) initiation of the acrosome reaction of the spermatozoon; (iii) prevention of polyspermy after initial entry of spermatozoa into the egg and subsequent exocytosis of cortical granule contents; (iv) maintenance of the integrity of the early embryo during the first few cleavage divisions before the development of tight junctions between the blastomeres and the occurrence of compaction; ( $v$ ) prevention of the attachment of the blastomeres to the epithelial lining of the oviduct during embryonic transit (Yanagimachi, 1994); and (vi) putative protection of the early embryo from potential immunological rejection (Ozgur et al., 1998).

In marsupials, far less is known about the functions of the zona pellucida and what data there are indicate that some of the functions differ from those of eutherian mammals. For instance, in contrast to eutherians, it appears that the prevention of polyspermy after fertilization may result from the deposition of a mucoid coat around the outer surface of the zona pellucida during oviduct transit (Rodger and Bedford, 1982; Selwood, 1992), with a consequence that changes in the zona pellucida resulting from a cortical granule exocytosis may not be required to prevent polyspermy. In addition, since the marsupial embryo becomes surrounded by a mucoid coat, this coat, rather than the zona pellucida, is likely to prevent embryonic attachment to the oviductal epithelium. In contrast to the eutherian zona pellucida, the zona pellucida of dasyurids and possums appears to facilitate the initial blastomere attachment during early embryogenesis, before the development of cell-cell adhesion mechanisms (Selwood, 1992, 2000; Frankenberg and Selwood, 1998). There also appear to be structural differences between eutherian and marsupial zonae pellucidae, as the marsupial zona pellucida is much more easily solubilized by dilute proteases (Rodger and Bedford, 1982; Bedford, 1991; Bedford and Breed, 1994). Do these differences in function, thickness and solubility of the zona pellucida between marsupial and eutherian mammals reflect significant differences in either its polymeric organization or its biochemistry?

\section{Biochemical components and structural organization of the eutherian zona pellucida}

In eutherians, the thickness of the zona pellucida ranges from 7 to $25 \mu \mathrm{m}$ (Dunbar et al., 1991; Sinowatz et al., 2001) and the physical appearance of its inner and outer regions varies (Phillips and Shalgi, 1980; Dunbar et al., 1991, 1994) depending on the species. Despite this variation, little consideration has been given to differences in the chemical composition of the zona pellucida. Furthermore, there is evidence that, in some species, different components of the zona pellucida are synthesized by different cell populations. For instance, in mice, expression of the ZPA, ZPB and ZPC genes appears to be restricted to the germ cells (Wassarman, 1988; Epifano et al., 1995), whereas in rabbits, pigs, cows, cynomolgus monkeys and humans, one or more of the zona pellucida genes is also expressed in the 
surrounding granulosa cells during some stages of folliculogenesis (Dunbar et al., 1994; Grootenhuis et al., 1996; Kölle et al., 1996, 1998; Martinez et al., 1996; Prasad et al., 2000; Sinowatz et al., 2001).

Regardless of where the genes are expressed, zona pellucida polypeptide chains become extensively glycosylated before secretion. Early studies revealed a major discrepancy between the reported molecular weights of the polypeptides of the three zona pellucida glycoproteins as deduced from cDNA cloning and SDS PAGE. Extensive glycosylation of the polypeptides was therefore suggested (for reviews, see Wassarman, 1988, 1999; Yanagimachi, 1994; Rankin and Dean, 2000). Subsequent work on zonae pellucidae stained with lectins (Shalgi and Raz, 1997; Avilés et al., 1999, 2000) identified an abundance of glycoconjugates with both $O$ - and $\mathrm{N}$-linked oligosaccharides. Furthermore, lectin-gold labelling has been used to demonstrate that the distribution of the saccharides varies throughout the zona pellucida (Avilés et al., 2000). These oligosaccharides appear critically important for spermzona binding, and most data indicate primary binding of the spermatozoon to the surface ligands on the oligosaccharide component of zona pellucida glycoproteins rather than to the polypeptide chains (for example, Sinowatz et al., 1995; Dell et al., 1999; Töpfer-Petersen, 1999; Wassarman, 1999; Tulsiani and Abou-Haila, 2001). The oligosaccharides of the glycoproteins provide the interface for specific sperm-zona binding, as demonstrated in studies involving deglycosylation and inhibition of sperm-zona binding with sugars and lectins (Mori et al., 1993).

Once the glycoproteins have been synthesized and secreted, how do they become organized into the acellular coat that comes to surround the oocyte? Wassarman (1988) proposed that, at least for the laboratory mouse, repeating units of ZPA and ZPC are formed, giving rise to filaments with a structural periodicity of 14-15 nm, which are crosslinked by ZPB dimers forming a three-dimensional mesh. Currently, it is neither known in detail how the zona pellucida molecules are synthesized and secreted, nor how polymerization results in the formation of these filaments. Green (1997) suggested that either three-dimensional crosslinking occurs extracellularly some distance from the oocyte surface, or that assembly of the zona filaments takes place on the surface of the oolemma before secretion into the perivitelline space. The Wassarman model of the zona structural organization was constructed from data derived from laboratory mice and is supported by ultrastructural observations (Greve and Wassarman, 1985), as well as by the occurrence of equimolar ratios of ZPA and ZPC and their sequence of synthesis (Wassarman, 1988; Epifano et al., 1995; for review, see Green, 1997). Furthermore, gene 'knock-out' experiments in mice have added further support for this model since a homozygous deletion of either ZPC or ZPA resulted in a lack of zona formation (Liu et al., 1996; Rankin et al., 1996, 1999; Wassarman et al., 1999), whereas a zona pellucida, albeit with aberrant organization, did form in ZPB knock-out mice (Rankin et al., 1996, 1999;
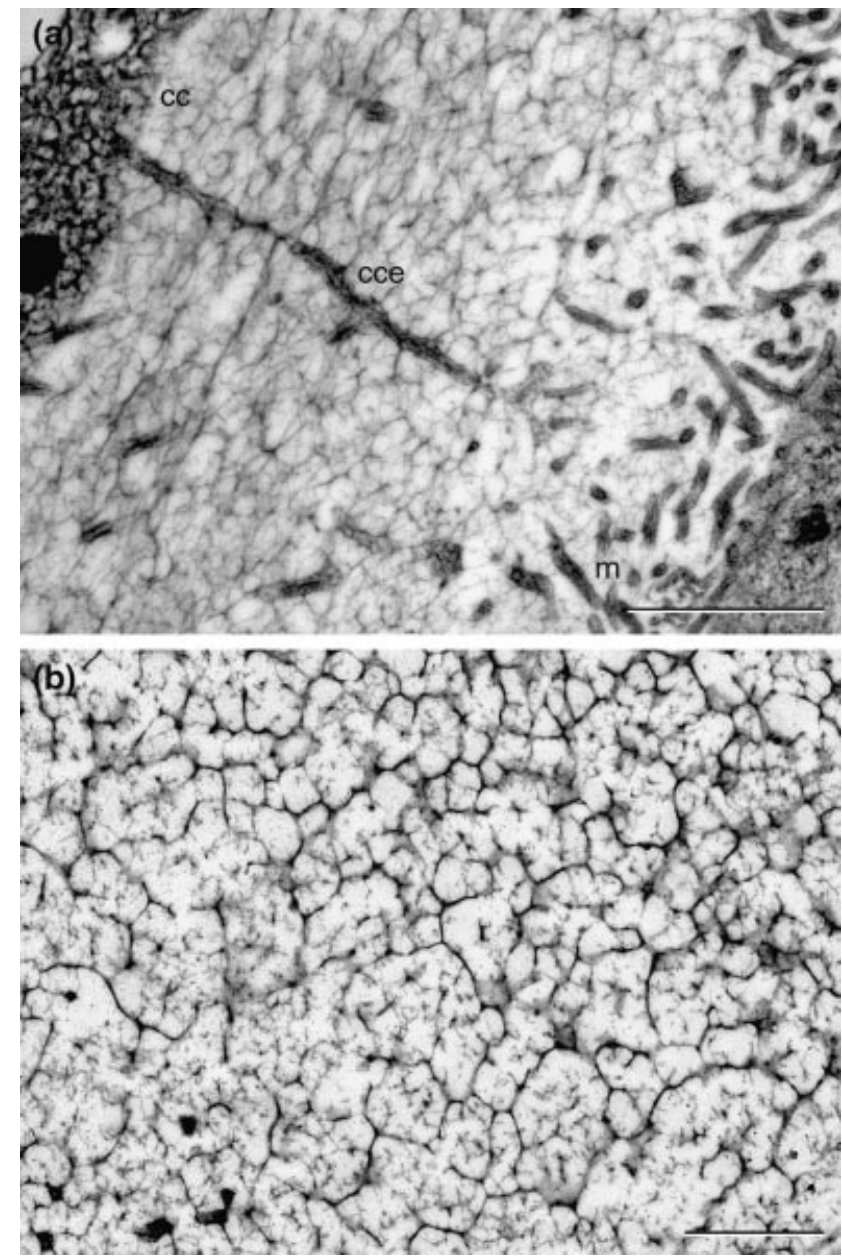

Fig. 3. Transmission electron microscopy of rapid frozen and freeze substitution preparations of brushtail possum zonae pellucidae obtained from around a preovulatory (a) and recently ovulated (b) possum (Trichosurus vulpecula) oocyte. Note the tightly ordered arrangement of filaments of the zona pellucida. Around the preovulatory oocyte close to the cumulus cell (cc) and cytoplasmic extension (cce), the filaments appear to be more linearly aligned than those near the microvilli $(\mathrm{m})$ of the oocyte. Scale bar represents $1 \mu \mathrm{m}$.

Rankin and Dean, 2000). Different molar ratios in other species, for example pigs, in which equivalent amounts of ZPB and ZPC and less ZPA occur (Nakano et al., 1996), together with the possibility of differences between human ZP1 and ZPB, indicate that the model proposed by Wassarman may not be applicable to the structural organization of the zona pellucida in species of other orders of mammals.

\section{Biochemical components and structural organization of the marsupial zona pellucida}

Low-power transmission and scanning electron microscopy (Breed, 1996; Fig. 2) indicate that the marsupial zona pellucida has a similar overall structure to that of eutherian 


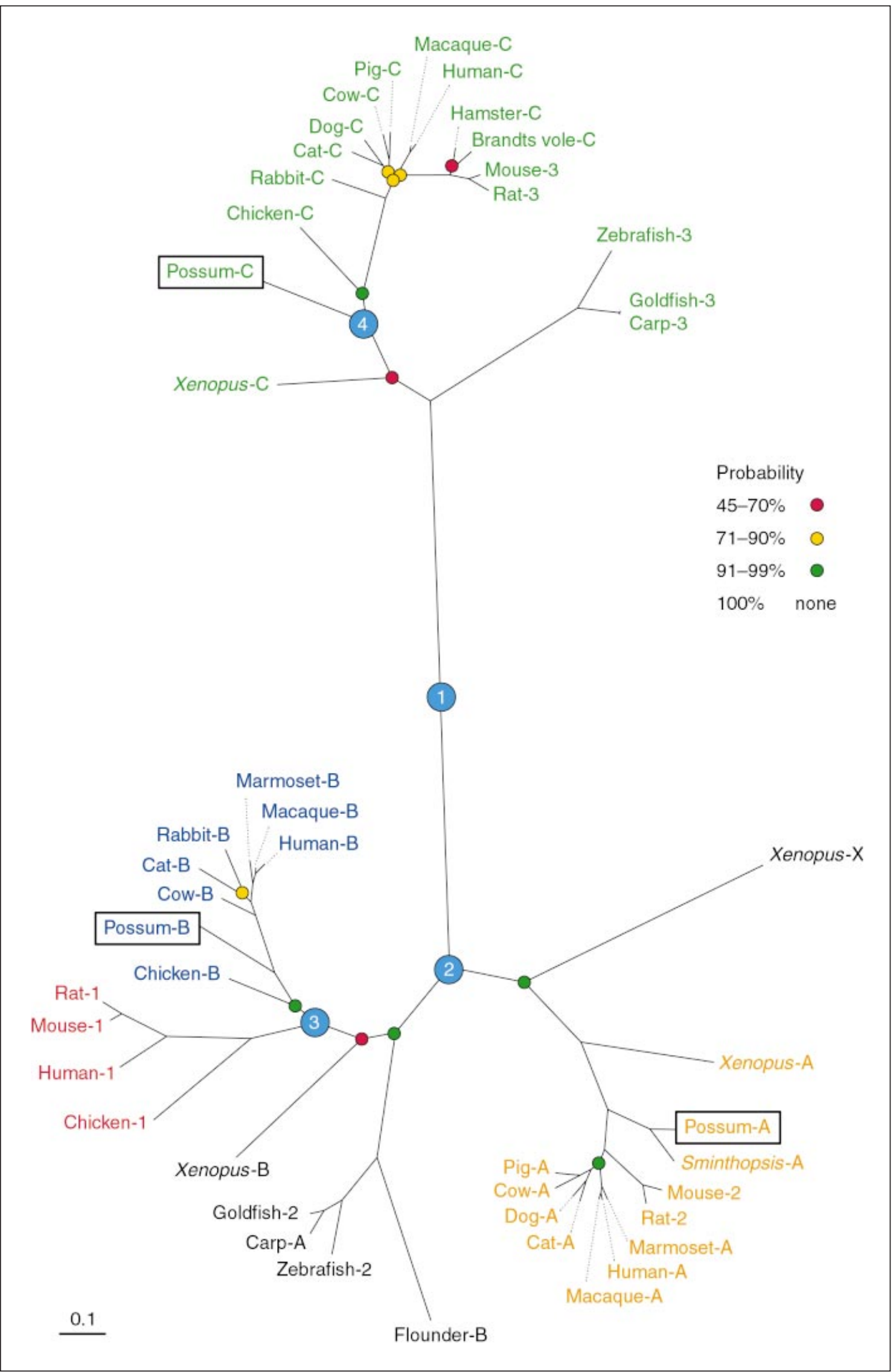

Fig. 4. For legend see facing page. 
mammals. Nevertheless, as previously indicated, in comparison with the eutherian zona pellucida, the marsupial zona pellucida responds differently to dilute proteases, and also appears to perform somewhat different functions. How do its chemical composition and detailed structural organization compare?

\section{Polypeptides}

Three cDNAs encoding zona pellucida from the brushtail possum, Trichosurus vulpecula, have been cloned and sequenced (Mate and McCartney, 1998; Haines et al., 1999; McCartney and Mate, 1999; Voyle et al., 1999). A comparison of the aligned polypeptide sequences with those from eutherians has revealed several notable similarities and few discernible differences. As with all zona pellucida sequences from amphibians, birds and eutherian mammals, each of the marsupial zona pellucida glycoproteins has an $\mathrm{N}$ terminal signal peptide and a C-terminal transmembrane domain. All zona pellucida sequences also have a highly conserved zona pellucida motif, which is likely to be essential for the formation of its filamentous structure. The ZPB glycoprotein has, in addition, a trefoil domain upstream from the zona pellucida motif, which may be involved in cross-linking the polypeptide chains. The distribution of cysteine residues in the zona pellucida polypeptides is highly conserved throughout marsupial and eutherian mammals. However, the extent of disulphide bonding in marsupial zonae pellucidae may differ from that in eutherians since there was a reduced susceptibility of the marsupial zona pellucida to disulphide reduction by $\beta$ mercaptoethanol and dithiothreitol (Chapman et al., 2000a). Although some conservation of the glycosylation sites is evident, there is considerable heterogeneity in the number and position of these sites (Mate and McCartney, 1998; Haines et al., 1999; Voyle et al., 1999). In addition, potential polymorphic variation has been found to occur in the ZPA of the brushtail possum (Voyle et al., 1999), and six non-synonymous substitutions occur outside the highly conserved domain; intraspecific variability in fertility may be associated with this variation.
In situ hybridization, using histological sections of possum ovaries, has shown that ZPA and ZPB expression appears to be restricted to the oocyte, at least within the limits of the colorimetric detection method. Furthermore, both mRNA transcripts appear to be present in the cytoplasm of at least some of the oocytes of primordial follicles of adult possums. Maximum staining for transcripts occurred in the oocytes of primary follicles in which there was a single layer of cuboidal follicular cells (Haines et al., 1999; Voyle et al., 1999), and in which some synthesis and secretion of zona pellucida extracellular matrix has already taken place (Breed, 1996; Mate, 1998). This finding indicates that a large amount of transcription and translation of the zona pellucida transcripts occurs in oocytes at an early stage of follicular development but that deposition of the matrix does not take place until differentiation of a primary follicle occurs.

\section{Glycoconjugates}

As in eutherian mammals, the co- and post-translational modifications to zona pellucida proteins are likely to play a significant role in the form and function of the zona pellucida in marsupials. The $\mathrm{N}$ - and $\mathrm{O}$-linked oligosaccharides of the eutherian zona pellucida probably facilitate its flexibility, longevity, as well as acting as ligands for sperm receptors and protecting the oocyte and early embryo (Green, 1997). Detailed characterization of the oligosaccharides of the eutherian zona pellucida has been carried out in pigs (for review, see Nakano and Yonezawa, 2001), cows (for example, Katsumata et al., 1996) and laboratory rodents (for example, Noguchi and Nakano, 1993; Easton et al., 2000). However, such characterization of zona pellucida glycoproteins is not technically possible at present for marsupial zonae owing to the limited availability of material. This constraint restricts investigation of the contribution of specific sugars in the oligosaccharides of the marsupial zona pellucida glycoproteins to lectin histochemistry (Chapman et al., 2000b) similar to that carried out in earlier studies on eutherian zonae pellucidae (Skutelsky et al., 1994; Parillo et al., 1996).

Fig. 4. Unrooted phylogeny based on aligned zona pellucida domain cDNA sequences from representative zona pellucida genes. The phylogeny shows that there are at least four paralogous groups of zona pellucida genes (ZPA, ZPB, ZP1 and ZPC), requiring a minimum of three gene duplication events (indicated at positions 1, 2 and 3). The ZPA genes are highlighted in yellow, the ZPB genes in blue, the ZP1 genes in red, and the ZPC genes in green. Duplications are identified by blue circles. Duplication 1 gave rise to the ancestral ZPC and the ancestral precursor of ZP1, ZPA and ZPB; this event pre-dated the divergence of amniotes from fish. Duplication 2 gave rise to the ancestral ZPA and the ancestral precursor of ZP1 and ZPB; this event pre-dated the divergence of reptiles, birds, and mammals. Duplication 3 gave rise to the ancestral ZP1 and ZPB gene lineages; this event also pre-dated the divergence of reptiles (including birds) and mammals. A fourth duplication is included to explain the anomalous position of possum ZPC. Zona pellucida domain protein sequences were aligned using CLUSTALW (Thompson et al., 1994), and then converted to cDNA (maintaining the alignment) for phylogenetic analysis. The tree was generated using the program BAMBE (Simon and Larget, 2000). The final tree is a consensus compiled from the results generated from four different random starts. The log likelihoods of trees sampled during each of the four runs converged, supporting the contention that the final tree belongs to a true maximum, not a local maximum. A total of $1.5 \times 10^{6}$ sampling iterations were included in the final summation. Posterior probabilities are indicated by the colour scheme shown in the key. The probability associated with duplications 1,2 and 3 is $100 \%$, and with duplication 4 is $97 \%$. Branch lengths are drawn to scale and indicate the rate of sequence change. Maximum parsimony with bootstrapping was also used to find a consensus tree for the same dataset. The branching order at crucial nodes was identical to that shown in the BAMBE consensus tree. 
Our studies of the marsupial zona pellucida have involved incubation of ovarian sections with either neuraminidase to remove masking sialic acids, or saponification to remove $\mathrm{O}$-acetyl groups, before staining with fluorescein-conjugated lectins. Although interspecific variation in glycoconjugate content was evident, its composition was in general similar to that of eutherian zonae pellucidae (Chapman et al., 2000b). Much of the glycoconjugate variation among marsupial species appears to relate to the $\mathrm{N}$-linked oligosaccharides and, in particular, to mannose. All of the seven marsupial species examined, each of which belongs to a different family, had mannose present in the zona pellucida (Chapman et al., 2000b), but in the dunnart (Sminthopsis crassicaudata), opossum (Monodelphis domestica), brushtail possum (Trichosurus vulpecula) and kangaroo (Macropus giganteus), the unmasking of $O$-acetyl groups was necessary before the mannose could be demonstrated. The fact that mannose residues are likely to be contained within $\mathrm{N}$-linked oligosaccharides is supported by the sequence data from the possum showing eight potential $N$-glycosylation sites for ZPA, seven for ZPB and two in ZPC after discounting one in each of ZPA and ZPC in which proline occupies the $X$ position of the determining sequon $\mathrm{N}-\mathrm{X}-\mathrm{S} / \mathrm{T}$. The sequon $\mathrm{N}-$ $\mathrm{P}-\mathrm{S} / \mathrm{T}$ is counter-indicated for $\mathrm{N}$-glycosylation, whereas conversely, the presence of serine as well as threonine (fifth site in both ZPA and ZPB of the possum) in the X position provides up to $95 \%$ efficiency for $N$-glycosylation (ShakinEshleman et al., 1996).

Identification and localization of the disaccharide $\beta(1-4)$ $\mathrm{N}$-acetyl-D-glucosamine in marsupial zona pellucida indicates conservation of this oligosaccharide in the zona pellucida throughout mammals. In the marsupial zona pellucida, as in that of eutherians (Avilés et al., 1994; Skutelsky et al., 1994), strong-to-intense binding of the lectin WGA is localized to the inner and outer regions. However, after saponification, this localization disappears, with strong-to-intense staining present throughout the zona pellucida. This staining pattern indicates a greater abundance of $\mathrm{O}$-acetylated sialic acid in the middle compartment of the marsupial zona pellucida compared with that in the inner and outer zones. It is possible that the function of these $O$-acetylated sialic acids is to inhibit degradation of glycoconjugates, since the presence of an acetyl group at the $0-4$ position blocks the action of neuraminidase (Schauer, 1988). The presence of sialic acid may prevent additional degradation of the internal sugar residues which, in turn, may prevent degradation of the protein backbone, as the more glycosylated a protein, the more resistant it is to enzymatic digestion (Semino et al., 1985; Jentoft, 1990). Although the presence of $O$-acetylated sialic acids in the central region of the zona pellucida of marsupials may help indirectly to maintain the integrity of the egg, the lack of $O$ acetylated sialic acids in the inner and outer regions may allow for selective glycolysis and proteolysis after an acrosome reaction or cortical granule content release.

Whether the differences in glycoconjugate composition of marsupial zona pellucida relate to the specificity of sperm binding to the zona pellucida is unknown. At present, this information is difficult to obtain because sperm-zona pellucida binding and penetration has been obtained only recently in vitro in an Australian species (Mate et al., 2000). This limitation has prevented the use of competitive binding assays using various carbohydrates to determine the sugar residues involved in sperm-zona pellucida binding. Furthermore, sperm-zona pellucida binding and penetration appears to take place only after spermatozoa have been incubated in oviduct-conditioned media, indicating an oviductal factor, or factors, that facilitate sperm-egg interactions (Sidhu et al., 1999). In the amphibian, Xenopus laevis, a trypsin-like oviduct factor, oviductin, transforms the egg envelope into a fertilizable vitelline envelope, by the cleaving of part of the C-terminus of the ZPC homologue gp43 into gp41 (Kubo et al., 1999; Lindsay et al., 1999). As the brushtail possum zona pellucida may change from a 'thick and diffuse' appearance before ovulation, to a 'thin and compact' appearance afterwards (Rodger and Mate, 1993), an analogous situation may occur in marsupials as well.

The highly glycosylated nature of the marsupial zona pellucida also indicates that it is extremely hydrated. Processing for electron microscopy may result in shrinkage of the zona pellucida, and lead to the occurrence of artefacts during the preparative process. Rapid freezing and freeze substitution are being used to overcome this problem and preserve the possum zona pellucida as close to the natural state as possible (J. A. Chapman, O. W. Wiebkin and W. G. Breed, unpublished). When prepared in this way and observed by routine transmission electron microscopy, the possum zona pellucida appeared to have a highly ordered, branched and organized pattern (Fig. 3). This regular structural organization did not reveal any noticeable inner and outer variation in the zona pellucida around ovulated oocytes as in the eutherian zona pellucida (Phillips and Shalgi, 1980; Dunbar et al., 1994; Yanagimachi, 1994). However, compared with the zona pellucida of ovulated eggs, the possum zona pellucida around intraovarian oocytes appears less well organized, possibly due to the traversing cytoplasmic extensions of cumulus cells with the material close to the somatic cells appearing more fibrillar than it does elsewhere (Fig. 3). The marked change in consistency of the possum zona pellucida around the time of ovulation may reflect a reorganization of the matrix as a result of the withdrawal of the cumulus cell extensions that takes place at this time.

\section{Evolution of the zona pellucida genes}

Questions about the orthology of the rodent and human ZPB genes have arisen from the low sequence identity observed between the corresponding CDNAs and proteins. A putative cDNA named ZP1, thought to represent the orthologue of mouse ZPB, has been identified in humans (Hughes and Barratt, 1999). The expression pattern of 
human ZP1, and the structure and function of its putative protein product, remain to be elucidated. Nevertheless, it seems likely that there are at least four paralogous groups of zona pellucida glycoprotein genes (that is, genes that trace their common ancestry back to gene duplication events) in amniotes, not all of which may contribute to the zona pellucida (Hughes and Barratt, 1999; Bausek et al., 2000). These findings can be explained by the hypothesis that, during the evolution of rodents, the ZPB gene was functionally replaced by the orthologue of the human ZP1 gene. It is unlikely that this event pre-dated the divergence of rodents from other eutherian orders, since rodents appear to be the only group of eutherian mammals in which this substitution occurred. Differences in the structure and function of ZP1 and ZPB may warrant a reappraisal of the degree to which human and mouse zona pellucida can be compared.

Several previous studies have inferred phylogenetic relationships among the various zona pellucida genes from sequence comparisons performed on the basis of per cent identity at the DNA or protein level. Such approaches can give misleading results as they assume that the relevant molecules evolve in a clock-like fashion. The evolution of the zona pellucida genes has been re-examined using the Monte Carlo Markov Chain (MCMC) convergence technique in the program BAMBE (Bayesian Analysis in Molecular Biology and Evolution - Version 2.02) (Simon and Larget, 2000), which enables the calculation of Bayesian posterior probabilities for each branch on the phylogeny. ZPA, ZPB and ZPC CDNA sequences from the brushtail possum (Mate and McCartney, 1998; Haines et al., 1999; McCartney and Mate, 1999; Voyle et al., 1999) were included in the analysis, as well as sequences from a range of eutherian mammals, birds and fish, all of which are present in the public domain on GenBank.

The resultant phylogeny (Fig. 4) has three important features. First, possum ZPA forms a monophyletic group with the eutherian ZPA genes, indicating that these genes share an orthologous relationship. Second, in the group of genes identified previously as ZPC, possum ZPC falls outside the chicken and eutherian ZPC lineage. One explanation for this could be that the possum ZPC gene is paralogous to the ZPC of birds and eutherian mammals, that is, it arose by gene duplication (see position 4, Fig. 4). Third, the rodent ZPB genes, together with the human (Hughes and Barratt, 1999) and chicken (Bausek et al., 2000) ZP1 genes, form a monophyletic group that is distinct from a group comprising chicken ZPB, possum ZPB and the other eutherian $\mathrm{ZPB}$ genes. This result indicates that these groups are paralogous and resulted from a gene duplication as indicated (see position 3, Fig. 4). Although the system of nomenclature for the zona pellucida genes is confusing, it is clear that within the existing framework, the possum ZPA and ZPB genes have been named according to their orthologous counterparts in eutherian mammals.

The research on the marsupial zona pellucida from the authors' laboratories was, in part, funded by grants from the Australian
Research Council. The authors thank Chris Leigh for his expert technical assistance, and Esther Breed for assistance with typing the manuscript.

\section{References}

Key references are identified by asterisks.

Avilés M, Martínez-Manárguez JA, Castells MT, Madrid JF and Ballesta J (1994) Cytochemical characterization of oligosaccharide side chains of the glycoproteins of rat zona pellucida: an ultrastructural study Anatomical Record 239 137-149

Avilés M, Castells MT, Abascal I, Martínez-Manárguez JA, Draber P, Kan FWK and Ballesta J (1999) Cytochemical localisation of GalNAc and GalNAc $\beta 1,4$ Gal $\beta 1$, 4 disaccharide in mouse zona pellucida Cell and Tissue Research 295 269-277

Avilés M, Okinaja T, Shur BD and Ballesta J (2000) Differential expression of glycoside residues in the mammalian zona pellucida Molecular Reproduction and Development 57 296-308

Bausek N, Waclawek W, Schneider WJ and Wohlrab F (2000) The major chicken egg envelope protein ZP1 is different from ZPB and is synthesised in the liver Journal of Biological Chemistry 27528 866-28 872

Bedford JM (1991) The coevolution of mammalian gametes. In $A$ Comparative Overview of Mammalian Fertilization pp 3-35 Eds BS Dunbar and MG O'Rand. Plenum Press, New York

Bedford JM (1998) Mammalian fertilization misread? Sperm penetration of the eutherian zona pellucida is unlikely to be a lytic event Biology of Reproduction 59 1275-1287

Bedford JM and Breed WG (1994) Regulated storage and subsequent transformation of spermatozoa in the fallopian tubes of an Australian marsupial, Sminthopsis crassicaudata. Biology of Reproduction $\mathbf{5 0}$ 845-854

Breed WG (1996) Egg maturation and fertilization in marsupials Reproduction, Fertility and Development 8 617-643

Chapman JA, Wiebkin OW and Breed WG (2000a) Structure of the zona pellucida of a model marsupial, the brushtail possum (Trichosurus vulpecula: Family Phalangeridae) Journal of Reproduction and Fertility Abstract Series 25 Abstract 6

*Chapman JA, Wiebkin OW and Breed WG (2000b) Interspecific variation of zona pellucida glycoconjugates in several species of marsupial Journal of Reproduction and Fertility 119 111-120

Dell A, Morris HR, Easton RL, Patankar M and Clark GF (1999) The glycobiology of gametes and fertilization Biochemica et Biophysica Acta 1473 196-205

Dunbar BS, Prasad SV and Timmons TM (1991) Comparative structure and function of mammalian zonae pellucidae. In A Comparative Overview of Mammalian Fertilization pp 97-114 Eds BS Dunbar and MG O'Rand. Plenum Press, New York

Dunbar BS, Avery S, Lee V, Prasad S, Schwahn N, Schwoebel E, Skinner S and Wilkins B (1994) The mammalian zona pellucida: its biochemistry, immunochemistry, molecular biology and developmental expression Reproduction, Fertility and Development 6 331-347

Easton RL, Patankar MS, Lattanzio FA, Leaven TH, Morris HR, Clark GF and Dell A (2000) Structural analysis of murine zona pellucida glycans Journal of Biological Chemistry 275 7731-7742

Epifano O, Liang L-F, Familari M, Moos MC, Jr and Dean J (1995) Coordinate expression of the three zona pellucida genes during mouse oogenesis Development 121 1947-1956

Frankenberg S and Selwood L (1998) An ultrastructural study of the role of an extracellular matrix during normal cleavage in a marsupial, the brushtail possum Molecular Reproduction and Development $\mathbf{5 0}$ 420-433

Green DPL (1997) Three-dimensional structure of the zona pellucida Reviews of Reproduction 2 147-156

Greve JM and Wassarman PM (1985) Mouse egg extracellular coat is a matrix of interconnecting filaments possessing a structural repeat Journal of Molecular Biology 181 253-264

Grootenhuis AJ, Philipsen HLA, de Breet-Grijsbach JTM and van Duin M (1996) Immunocytochemical localisation of ZP3 in primordial follicles 
of rabbit, marmoset, rhesus monkey, and human ovaries using antibodies against human ZP3 Journal of Reproduction and Fertility Supplement 50 43-54

*Haines BP, Rathjen PD, Hope RM, Whyatt LM, Holland MK and Breed WG (1999) Isolation and characterisation of a cDNA encoding a zona pellucida protein ZPB from the marsupial Trichosurus vulpecula (brushtail possum) Molecular Reproduction and Development 52 174-182

Harris JD, Hibler DW, Fontenot GK, Hsu KT, Yurewicz EC and Sacco AG (1994) Cloning and characterisation of the zona pellucida gene and cDNAs from a variety of mammalian species: the ZPA, ZPB and ZPC gene families DNA Sequence 4 361-393

*Hughes DC and Barratt CLR (1999) Identification of the true human orthologue of the mouse ZP1 gene: evidence for greater complexity in the mammalian zona pellucida? Biochimica et Biophysica Acta 1447 303-306

Jentoft N (1990) Why are proteins O-glycosylated? Trends in Biochemical Science 15 291-294

Katsumata T, Noguchi S, Yonezawa N, Tanokura M and Nakano M (1996) Structural characterization of the $\mathrm{N}$-linked carbohydrate chains of the zona pellucida glycoproteins from bovine ovarian and fertilized eggs European Journal of Biochemistry 240 448-453

Kölle S, Sinowatz F, Boie G, Totzauer I, Amselgruber W and PlendI J (1996) Localization of the mRNA encoding the zona pellucida ZP3 $\alpha$ in the porcine ovary, oocyte, and embryo by nonradioactive in situ hybridization Histochemical Journal 28 441-447

Kölle S, Sinowatz F, Boie G and Palma G (1998) Differential expression of ZPC in the bovine ovary, oocyte and embryo Molecular Reproduction and Development 49 435-443

Kubo H, Matsushita M, Kotani M, Kawasaki H, Saido TC, Kawashima S, Katagiri C and Suzuki A (1999) Molecular basis for oviductin-mediated processing from gp43 to gp41, the predominant glycoproteins of Xenopus egg envelopes Developmental Genetics 25 123-129

Lindsay LL, Wieduwilt MJ and Hedrick JL (1999) Oviductin, the Xenopus laevis oviductal protease that processes egg envelope glycoprotein gp43, increases sperm binding to envelopes and is translated as part of an unusual mosaic protein composed of two proteases and several CUB domains Biology of Reproduction 60 989-995

Liu C, Litscher ES, Mortillo S, Sakai Y, Kinloch RA, Stewart CL and Wassarman PM (1996) Targeted disruption of the mZP3 gene results in production of eggs lacking a zona pellucida and infertility in female mice Proceedings of the National Academy of Sciences 93 5431-5436

* McCartney CA and Mate KE (1999) Cloning and characterisation of a zona pellucida 3 cDNA from a marsupial, the brushtail possum Trichosurus vulpecula. Zygote 7 1-9

Martinez ML, Fontenot GK and Harris JD (1996) The expression and localization of zona pellucida glycoproteins and mRNA in cynomolgus monkeys (Macaca fascicularis) Journal of Reproduction and Fertility Supplement 50 35-41

Mate KE (1998) Timing of zona pellucida formation in the tammar wallaby (Macropus eugenii) and brushtail possum (Trichosurus vulpecula) Animal Reproduction Science 53 237-252

Mate KE and McCartney CA (1998) Sequence and analysis of a zona pellucida 2 cDNA (ZP2) from a marsupial, the brushtail possum, Trichosurus vulpecula. Molecular Reproduction and Development 51 322-329

Mate KE, Sidhu KS, Molinia FC, Glazier AM and Rodger JC (2000) Sperm binding and penetration of the zona pellucida in vitro but not sperm-egg fusion in an Australian marsupial, the brushtail possum (Trichosurus vulpecula) Zygote 8 189-196

Mori K, Daitoh T, Kamada M, Maeda N, Maegawa M, Hirano K, Irahara M and Aono T (1993) Blocking of fertilization by carbohydrates Human Reproduction 8 1729-1732

Nakano M and Yonezawa N (2001) Localization of sperm ligand carbohydrate chains in pig zona pellucida glycoproteins Cells, Tissues, Organs 168 65-75

Nakano M, Yonezawa N, Hatanaka Y and Noguchi S (1996) Structure and function of the $\mathrm{N}$-linked carbohydrate chains of pig zona pellucida glycoproteins Journal of Reproduction and Fertility Supplement $\mathbf{5 0}$ 25-34
Noguchi S and Nakano M (1993) Structural characterization of the $N$-linked carbohydrate chains from mouse zona pellucida glycoproteins ZP2 and ZP3 Biochemica et Biophysica Acta 1158 217-226

Ozgur K, Patankar M, Oehninger S and Clark GF (1998) Direct evidence for the involvement of carbohydrate sequences in human sperm-zona pellucida binding Molecular Human Reproduction 4 318-324

Parillo F, Stradaioli G, Dall'Aglio C and Verini-Supplizi A (1996) Characterization of the complex carbohydrates in the zona pellucida of mammalian oocytes using lectin histochemistry Veterinary Research Communications $20225-236$

Phillips DM and Shalgi RM (1980) Surface properties of the zona pellucida Journal of Experimental Zoology 213 1-8

Prasad SV, Skinner SM, Carino C, Wang N, Cartwright J and Dunbar BS (2000) Structure and function of the proteins of the mammalian zona pellucida Cells, Tissues, Organs 166 148-164

Rankin T and Dean J (2000) The zona pellucida: using molecular genetics to study the mammalian egg coat Reviews of Reproduction 5 114-121

Rankin T, Familari M, Lee E, Ginsberg A, Dwyer N, Blanchette-Mackie J, Drago J, Westphal H and Dean J (1996) Mice homozygous for an insertional mutation in the ZP3 gene lack a zona pellucida and are infertile Development 122 2903-2910

Rankin T, Talbot P, Lee E and Dean J (1999) Abnormal zonae pellucidae in mice lacking ZP1 result in early embryonic loss Development 126 3847-3855.

Rodger JC and Bedford JM (1982) Separation of sperm pairs and sperm-egg interactions in the opossum Didelphis virginiana. Journal of Reproduction and Fertility 64 171-179

Rodger JC and Mate KE (1993) Marsupial gametes and fertilization Today's Life Science 50 28-31

Schauer R (1988) Sialic acids as antigenic determinants of complex carbohydrates. In The Molecular Immunology of Complex Carbohydrates pp 47-72 Eds AM Wu and LG Adams. Plenum Press, New York

Selwood L (1992) Mechanisms underlying the development pattern in marsupial embryos. In Current Topics in Developmental Biology pp 175-233 Ed. RA Pedersen. Academic Press, New York

Selwood L (2000) Marsupial egg and embryo coats Cells, Tissues, Organs $166208-219$

Semino GA, Restani P and Cerletti P (1985) Effect of bound carbohydrate on the action of trypsin on lupin seed glycoproteins Journal of Agricultural Food Chemistry 33 196-199

Shakin-Eshleman SH, Spitalnik SL and Kasturi L (1996) The amino acid at the $\mathrm{X}$ position of an Asn-X-Ser sequon is an important determinant of $\mathrm{N}$ linked core-glycosylation efficiency Journal of Biological Chemistry 271 6363-6366

Shalgi $\mathbf{R}$ and $\mathbf{R a z} \mathbf{T}$ (1997) The role of carbohydrate residues in mammalian fertilization Histology and Histopathology 12 813-822

Sidhu KS, Mate KE, Molinia FC, Glazier AM and Rodger JC (1999) Secretory proteins from the female reproductive tract of the brushtail possum (Trichosurus vulpecula): binding to sperm and effects on sperm survival in vitro. Reproduction, Fertility and Development 11 329-336

Simon D and Larget B (2000) Bayesian Analysis in Molecular Biology and Evolution (BAMBE) version 2.02 beta. Department of Mathematics and Computer Science, Duquesne University

Sinowatz F, Amselgruber W, Töpfer-Petersen E, Totzauer E, Calvete K and Plendl J (1995) Immunocytochemical characterization of porcine zona pellucida during follicular development Anatomy and Embryology (Berlin) 191 41-46

Sinowatz F, Kölle S and Töpfer-Petersen E (2001) Biosynthesis and expression of zona pellucida glycoproteins in mammals Cells, Tissues, Organs 168 24-35

Skutelsky E, Ranen E and Shalgi R (1994) Variations in the distribution of sugar residues in the zona pellucida as possible species-specific determinants of mammalian oocytes Journal of Reproduction and Fertility 100 35-41

Thompson JD, Higgins DG and Gibson TJ (1994) CLUSATL-W: improving the sensitivity of progressive multiple sequence alignment through sequence weighting, position-specific gap penalties, and weight matrix choice. Nucleic Acid Research 22 4673-4680

Töpfer-Petersen E (1999) Carbohydrate-based interactions on the route of a spermatozoon to fertilization Human Reproduction Update 5 314-329 
Tulsiani DRP and Abou-Haila A (2001) Mammalian sperm molecules that are potentially important in interacting with female genital tract and egg vestments Zygote 9 51-69

*Voyle RB, Haines BP, Loffler KA, Hope RM, Rathjen PD and Breed WG (1999) Isolation and characterisation of zona pellucida A(ZPA) cDNAs from two species of marsupial: regulated oocyte-specific expression of ZPA transcripts Zygote 7 239-248

Wassarman PM (1988) Zona pellucida glycoproteins Annual Review of Biochemistry 57 415-442
Wassarman PM (1999) Mammalian fertilization: molecular aspects of gamete adhesion, exocytosis and fusion Cel/ 96 175-183

Wassarman PM, Chen J, Cohen N, Litscher ES, Liu C, Qi H and Williams Z (1999) Structure and function of the mammalian egg zona pellucida Journal of Experimental Zoology 285 251-258

Yanagimachi R (1994) Mammalian fertilization. In The Physiology of Reproduction pp 189-317 Eds E Knobil and JD Neill. Raven Press, New York 